\title{
Prognostic implication of the loss of TGFBR2 expression in oral carcinoma
}

\author{
V. P. SIVADAS ${ }^{1}$, G. SAAKSHI ${ }^{2}$, E. M. IYPE ${ }^{3}$, A. BALAN ${ }^{2}$, S. KANNAN ${ }^{1, *}$ \\ ${ }^{1}$ Division of Cancer Research, Regional Cancer Centre, Thiruvananthapuram-695011, Kerala, India; ${ }^{2}$ Department of Oral Medicine and Radiology, \\ Government Dental College, Kozhikode, Kerala, India; ${ }^{3}$ Division of Surgical Oncology, Regional Cancer Centre, Thiruvananthapuram-695011, \\ Kerala, India
}

*Correspondence: kannans@rcctvm.gov.in

Received January 31, 2014 / Accepted November 7, 2014

\begin{abstract}
Oral squamous cell carcinoma (OSCC) is a disease that strikes many worldwide, accounting for more than 145,000 deaths annually. This study examined the role of Transforming Growth Factor Beta (TGF $\beta$ ) signalling alterations in oral carcinogenesis and also its influence on the disease prognosis. In presented study, we evaluated the protein-level alterations of core TGF $\beta$ signalling members in 20 potentially malignant oral disorders (PMDs) - leukoplakia \& submucous fibrosis and 87 oral cancer samples by Western blotting. Further, we analysed the association between these alterations and prognosis of oral carcinoma. For statistical analyses, univariate test like Student's ' $t$ '-test to compare expression level of various genes and logrank test has been used to compare the Kaplan-Meier survival curves. The multivariate model such as Cox's proportional hazard regression was used to verify the independent influence of each variable on the survival endpoints. A gradual decrease in the expression of TGF $\beta$ signalling members like SMAD2, SMAD4, TGFBR1 and TGFBR2 have been noted from normal to PMD in oral cancers. The bio-activeforms of SMAD2/3 also showed a similar trend. SMAD3 protein was downregulated significantly to the PMD stage itself. Thus an inverse correlation was observed between expression of TGF $\beta$ members and oral cancer progression. Furthermore, oral cancer patients showing TGFBR2 downregulation exhibited poor disease-free survival $(\mathrm{p}=0.005)$ and poor overall survival $(\mathrm{p}=0.012)$. Thus, assessing the TGFBR2 protein levels can serve as one of the prognostic marker for oral cancer.
\end{abstract}

Key words: oral cancer,TGFBR2 expression, prognosis, Western immunoblot

Oral cancers comprise a group of malignancies that develop on lips, tongue, gingiva, floor of mouth and other unspecified parts of the mouth namely buccal mucosa, retro-molar and vestibular area [1]. The most common type of oral cancer is squamous cell carcinoma (OSCC) than the verrucous and adeno-squamous cell carcinoma, it accounts for more than $90 \%$ of the oral cancers [2]. Annually, oral carcinoma accounts for $\sim 274,000$ cases and $\sim 145,000$ deaths, of which two-thirds occur in developing countries [3,4]. Tobacco and betel chewing are main cause for such high prevalence of oral cancer in developing countries [5] and HPV infection rate is comparatively low in these populations [6].

Despite the advances in cancer treatment, the five-year survival rate of oral carcinoma is less than 50\% throughout the world. Furthermore, this scenario has not improved over the past two decades [3,4]. Local and/or loco-regional recurrences are common in oral cancers, even in those patients who initially showed complete response to the treatment [7].
Thus, the oncologist has to bear many factors in mind while deciding and selecting the treatment protocol for oral cancer, as patients show varied response to each treatment plan which may be due to the differences in the molecular pathways altered during carcinogenesis. This scenario necessitates the development of molecular markers, which can divide patients into different molecular groups such that optimum treatments can be administered to each group.

TGF $\beta$ signalling is a well-known tumor suppressor signalling pathway. Binding of the TGF $\beta$ family of cytokines to the type II TGF $\beta$ Receptor (TGFBR2) can trigger the downstream signalling cascade. On ligand binding mediated activation, TGFBR2 forms heteromeric complex with type I TGF $\beta$ receptor (TGFBR1). The TGFBRI/RII receptor complex then conveys downstream signalling through the pathway consisted of SMADs, SMAD2 and SMAD3. They are commonly called receptor regulated SMADs or R-SMADs. The phosphorylated R-SMADs can form heteromeric complex with the common 
mediator SMAD (Co-SMAD), SMAD4 and this enables the nuclear translocation of the complex and the expression of various target genes [8].

TGF $\beta$ signalling cascade is often altered in epithelial malignancies. Loss of TGF $\beta$ tumor suppressor pathway members like SMAD4, TGFBR1 and TGFBR2 accounts for roughly $50 \%$ of the solid tumors of pancreas as well as colon $[9,10]$. In one of the earlier studies, Kim et al. (1996) described the inverse correlation between the loss of TGFBR1 and TGFBR2 expression and the tumor grade in human prostate cancer[11]. Their results indicate that human prostate cancer cells frequently show a gradual loss of TGF $\beta$ receptor expression during cancer progression. Hence, it is possible that studies regarding the expression alteration of TGF $\beta$ signalling in cancer may provide novel biomarkers for cancer therapy. In addition, studies on the relationship between TGF $\beta$ signaling alterations and oral cancer progression are lacking. Therefore, in this work, we have evaluated the association between protein level alterations of TGF $\beta$ signaling members such as SMAD2, SMAD3, SMAD4, TGFBR1 and TGFBR2 in PMD and OSCC cases by employing Western-immunoblotting technique. We have also analyzed the bioactive forms of SMAD proteins- pSMAD2 and pSMAD3 levels in these samples [8]. Further, we have assessed the relationship between these alterations and clinicobiological features of oral cancers. The result shows that TGFBR2 expression level has been reduced in $60 \%$ of oral cancers samples and downregulation of TGFBR2 is significantly associated with poor treatment response. Hence, assessing the expression pattern of TGFBR2 before treatment may be useful to know the aggressive nature of the tumor and also it could may help in the treatment planning.

\section{Materials and methods}

Sample collection, ethics statement, and follow-up. OSCC samples and normal oral mucosa samples were collected from the Head \& Neck Cancer Clinic of the Regional Cancer Centre, Thiruvananthapuram, India. The PMD samples and normal oral mucosa were collected from the Department of Oral Medicine and Radiology, Government Dental College, Kozhikode, India. Normal mucosa samples were collected from the buccal mucosa of healthy individuals visited the outpatient clinic for third molar extraction. All samples were collected after obtaining patient's written informed consent. This study was approved by the Institutional Review Board and the Human Ethics Committee. In total, 87 OSCCs, 20 PMDs and 25 normal samples were collected for the present study. The clinical staging of the patients followed the WHO criteria [12]. Standard treatment protocols were administered to the patients according to their tumor stage and individual clinical status, as we have detailed elsewhere [13]. After the treatment, all patients were actively followedup at respective clinics, at an interval of four to eight weeks for a minimum period of 36 months [13].

Western immuno-blotting. Approximately $50 \mu \mathrm{g}$ of total protein isolated from the tissue samples were separated on $10 \%$
SDS-PAGE gel and transferred to polyvinylidene difluoride membrane (Millipore,Billerica, MA, USA). The membrane was blocked using $5 \%$ of BSA (Santa Cruz Biotechnology, Inc., Dallas, TX, USA) and the membrane was incubated with corresponding primary antibodies for two and a half hours. Primary antibodies against $\beta$-Actin, SMAD4, TGFBR1, TGFBR2, pSMAD2/3 and secondary antibodies were purchased from (Santa Cruz Biotechnology, Inc., Dallas, TX, USA. Primary antibodies against SMAD2 and SMAD3 were purchased from (Imgenex India Pvt. Ltd., Bhubaneswar, Odisha, India. Horseradish peroxidase (HRP) conjugated secondary antibody and Super Signal West Pico Chemiluminescent Substrate kit (Pierce, Rockford, IL, USA) were used for detecting specifically bound primary antibodies. The immunoblots were documented in a Chemiluminescence cum Fluorescence Imaging System, FluorChem M (Protein Simple Inc., Santa Clara, California, USA).

Normalization of the data. The digitally acquired bands were quantified densitometrically using the Image J software (http://rsbweb.nih.gov/ij/) of National Institute of Health (NIH), Bethesda, Maryland, USA. In order to account for the variations arising from differences in protein load, we normalized the protein level expression data using $\beta$-Actin as loading control. The ratio between the intensity (densitometric value) of the band of interest (target protein) to loading control gene in the same sample has been calculated. In order to analyze the protein level expression variations of TGF $\beta$ members in oral carcinoma, the expression ratio TGF $\beta$ members in tumor samples were compared with the mean expression ratio of the same TGF $\beta$ member in normal samples. Altogether, 20 normal tissues were used for assessment. To reduce the bias, we considered $\pm 20 \%$ expression variations from the mean expression ratio in normal as unaltered category (in order to account for the person to person variations in gene expression).

Statistical analysis. The associations between the expression-level alterations of analysed TGF $\beta$ members and various clinico-pathological factors and survival endpoints were examined as we have explained elsewhere [13]. Student's t-test was employed for comparing the expression pattern of various proteins in carcinoma samples with normal mucosa samples. The Kaplan-Meier survival plot with the Log-Rank test was used to assess the association of expression-level alterations of various proteins with survival endpoints such a as diseasefree survival (DFS) and overall survival (OS). To assess the independent influence of factors that showed significance in the Kaplan-Meier survival analyses, the multivariate Cox's proportional hazard regression model was used. A two-sided p-value $<0.05$ was considered statistically significant unless otherwise noted.

\section{Results}

Disruption of TGF $\beta$ signaling is frequent in OSCCs. The clinico-pathological characteristics of the study cohort 
are given in Table 1 . The present study identified expression alteration of at least one TGF $\beta$ member evaluated in most of the OSCC samples, indicating the importance of TGF $\beta$ signal disruption during oral cancer progression. Notably, some of the analyzed OSCC samples showed SMAD3, SMAD4 and TGFBR2 expression as less as $10 \%$ of the normal samples

Table 1. Clinico-pathological characteristics of the study cohort

\begin{tabular}{lcc}
\hline Clinico-pathological features & OSCCs & PMDs \\
\hline All Cases & $\mathbf{8 7}$ & $\mathbf{2 0}$ \\
Sex & & \\
Male & $56(64.4 \%)$ & $10(50.0 \%)$ \\
Female & $31(35.6 \%)$ & $10(50.0 \%)$ \\
\hline Age (Mean age \pm SD) & $55.2 \pm 11.9$ & $48.9 \pm 13.6$ \\
\hline Site & & \\
Tongue & $40(46.0 \%)$ & $1(5.0 \%)$ \\
Buccal mucosa & $47(54.0 \%)$ & $19(95.0 \%)$ \\
\hline Habit & $12(13.8 \%)$ & $2(10.0 \%)$ \\
None & $28(32.2 \%)$ & $12(60.0 \%)$ \\
Chewing & $3(3.4 \%)$ & $1(5.0 \%)$ \\
Smoking & $5(5.7 \%)$ & $2(10.0 \%)$ \\
Alcohol & $10(11.5 \%)$ & $1(5.0 \%)$ \\
Chewing+Snuff & $12(13.8 \%)$ & $1(5.0 \%)$ \\
Smoking+Alcohol & $17(19.5 \%)$ & $1(5.0 \%)$ \\
Chewing+Smoking+Alcohol & &
\end{tabular}

\begin{tabular}{|c|c|c|}
\hline Histopathology & & \\
\hline $\begin{array}{l}\text { Well differentiated squamous cell } \\
\text { carcinoma (WDSCC) }\end{array}$ & $18(20.6 \%)$ & \\
\hline $\begin{array}{l}\text { Moderately differentiated squamous } \\
\text { cell carcinoma (MDSCC) }\end{array}$ & $61(70.1 \%)$ & \\
\hline $\begin{array}{l}\text { Poorly differentiated squamous cell } \\
\text { carcinoma (PDSCC) }\end{array}$ & $9(10.3 \%)$ & \\
\hline Early OSF & & $3(15.0 \%)$ \\
\hline Moderate OSF & & $3(15.0 \%)$ \\
\hline Advanced OSF & & $2(10.0 \%)$ \\
\hline Leukoplakia with Mild Dysplasia & & $5(25.0 \%)$ \\
\hline Leukoplakia with Moderate Dysplasia & & $4(20.0 \%)$ \\
\hline Leukoplakia with Severe Dysplasia & & $1(5.0 \%)$ \\
\hline Leukoplakia turning malignant & & $2(10.0 \%)$ \\
\hline T-Status & & \\
\hline T1 & $9(10.3 \%)$ & \\
\hline T2 & $29(33.3 \%)$ & \\
\hline T3 & $16(18.4 \%)$ & \\
\hline T4 & $31(35.6 \%)$ & \\
\hline Not Available & $2(2.3 \%)$ & \\
\hline N-Status & & \\
\hline No & $28(32.2 \%)$ & \\
\hline N1 & $29(33.3 \%)$ & \\
\hline N2 & $27(31.0 \%)$ & \\
\hline N3 & $1(1.1 \%)$ & \\
\hline Not Available & $2(2.3 \%)$ & \\
\hline Disease Stage (OSCCs) & & \\
\hline I & $6(6.9 \%)$ & \\
\hline II & $13(14.9 \%)$ & \\
\hline III & $21(24.1 \%)$ & \\
\hline IV & $45(51.7 \%)$ & \\
\hline Not Available & $2(2.3 \%)$ & \\
\hline
\end{tabular}

OSCC = Oral Squamous Cell Carcinoma; $\mathrm{PMD}=$ Potentially malignant oral disorders; OSF=Oral submucous fibrosis; $\mathrm{SD}=$ Standard deviation.
(Figure 1). As compared to normal oral mucosa samples, more than $60 \%$ of the OSCC cases showed diminished levels of SMAD2 and TGFBR2 protein, whereas decreased SMAD3 and SMAD4 protein levels were observed in roughly $50 \%$ of samples. However, in most of the samples the expression of TGFBR1 is almost same as in normal controls (Figure 1 \& Figure 2A). Notably, robust expression of bioactive forms of SMADs- pSMAD2 and pSMAD3 was found only in $44.8 \%$ and $36.7 \%$ of samples respectively, indicating disabling of TGF $\beta$ signalling is a common event in OSCCs (Table 2).

TGF $\beta$ members show a gradual decrease in expression from normal to OSCC through potentially malignant oral disorders. In order to get an idea about the role of these alterations in oral carcinogenesis, we evaluated the expression pattern of TGF $\beta$ members in premalignant oral lesions like oral submucous fibrosis (OSF) and leukoplakia. The mean protein levels of various TGF $\beta$ members in PMDs and OSCC with respect to normal mucosa is given in Table 3. Interestingly, all the analyzed genes except SMAD3 showed a gradual decrease of protein expression from normal to PMD to OSCCs (Figure 2A \& 2B), suggesting the possibility for the gradual silencing of TGF $\beta$ signaling during oral carcinogenesis. There was a significant difference between SMAD2 and TGFBR2 expression between PMD and OSCC cases $(p=0.015$ and $\mathrm{p}=0.014$ respectively). No statistically significant difference between PMDs and OSCCs was observed with reference to the expressions of other analyzed genes (Supplementary Figure 1 and Table 3).

The pSMAD/total SMAD ratio in PMDs and OSCCs are similar to normal. Further, we queried whether there is any deviation from the PSMAD to SMAD ratio in PMDs as well as in OSCCs as compared to the normal tissues. Interestingly, we did not observe any significant variation in this ratio (Figure $2 \mathrm{C}$ ). The PMD cases showed phospho-SMADs to total SMADs ratio was similar to that of normal tissue, whereas oral cancer cases showed slightly higher $\mathrm{pSMAD/SMAD}$ ratio. However, these values were not statistically significant.

Correlation between protein level alterations of TGF $\beta$ signalling members and clinical outcome in OSCCs. Analysis using Kaplan-Meier estimates revealed that the protein level alterations of TGFBR2 has a significant influ-

Table 2. The protein level expression patterns of TGF $\beta$ signaling members in OSCC samples.

\begin{tabular}{lccc}
\hline \multirow{2}{*}{ Protein } & \multicolumn{3}{c}{ Protein levels (Compared to Normal) } \\
\cline { 2 - 4 } & No change & Upregulation & Down regulation \\
\hline pSMAD2 & $27(31.0 \%)$ & $12(13.8 \%)$ & $48(55.2 \%)$ \\
pSMAD3 & $11(12.6 \%)$ & $21(24.1 \%)$ & $55(63.2 \%)$ \\
SMAD2 & $20(23.0 \%)$ & $7(8.04 \%)$ & $60(68.9 \%)$ \\
SMAD3 & $30(34.5 \%)$ & $11(12.6 \%)$ & $46(52.9 \%)$ \\
SMAD4 & $20(23.0 \%)$ & $21(24.1 \%)$ & $46(52.9 \%)$ \\
TGFBR1 & $32(36.8 \%)$ & $14(16.1 \%)$ & $41(47.1 \%)$ \\
TGFBR2 & $12(13.8 \%)$ & $14(16.1 \%)$ & $61(70.1 \%)$ \\
\hline
\end{tabular}




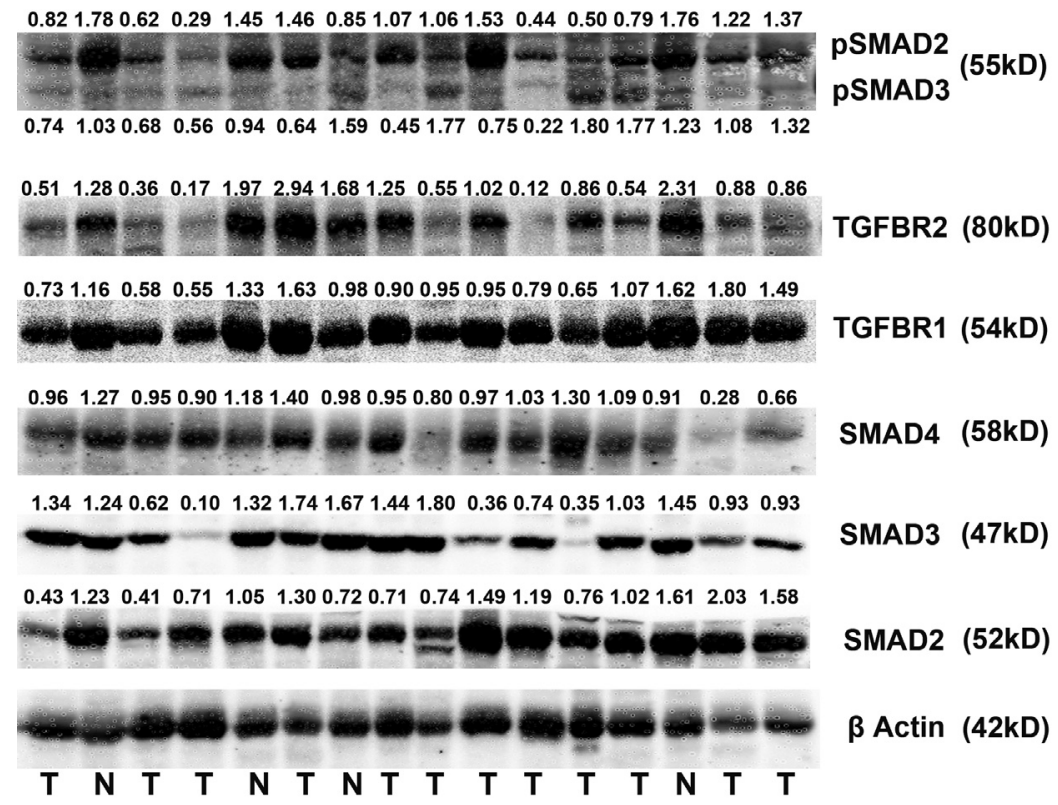

Figure 1. Western immuno-blot image showing protein level expression patterns of the analysed TGF $\beta$ pathway members in OSCCs. The $\beta$-Actin protein was used as loading control. The values given in the figure are normalized values using $\beta$-Actin. ( $T=$ Tumor; $N=N o r m a l)$.

ence on the disease-free survival ( $p$ value $=0.005$; Figure2D) as well as overall survival ( $\mathrm{p}$ value $=0.012$; Figure $2 \mathrm{E}$ ) of oral cancer patients. Interestingly, the protein levels of other genes including SMAD2 (which showed down regulation in a significant percentage of samples) did not show statistically significant association with oral cancer prognosis. However, there was a five-fold increase in the relative risk of reduced overall survival in SMAD2 down regulated cases. Indeed, the well-known clinical variables $\mathrm{T}$-status, $\mathrm{N}$-status and stage of disease were also found to significantly influence the diseasefree survival in the univariate analysis (DFS: $\mathrm{p}$ values 0.002 , $0.004 \& 0.001$ respectively and OS: $p$ values $<0.001$ for all three variables). Multivariate analysis using Cox's regression model revealed that TGFBR2 protein levels have an independent influence on both the disease-free survival as well as overall survival of the patients ( $p$ values $D F S=0.020$ \& OS= 0.025; Table 4). Among clinical factors, only 'stage of the disease' is found to have an independent prognostic effect (Supplementary Table 1).

\section{Discussion}

Loss of TGF $\beta$ responsiveness is a hallmark of epithelial malignancies. In addition to mutations, epigenetic alterations of this pathway are reported in a variety of cancers including HNSCCs [9]. Hence, the present study analyzed protein level alterations in TGF $\beta$-SMAD signalling members in oral cancer samples and attempted to delineate the correlation between these alterations and disease progression. We found that, as compared to normal, the expression of TGF $\beta$ members is lower in PMDs and least in OSCCs. This indicates possibility of gradual silencing of this tumor suppressor pathway during the progression from normal to PMDs to OSCCs. Previously, we have shown that mutations form only part of the molecular alterations of this pathway in OSCCs and occur mostly in Stages 3 \& 4 OSCCs [14]. This suggests the involvement of some other mechanisms like promoter methylation ormiRNA-mediated silencing of this pathway during oral cancer progression. Notably, many authors have clearly shown that methylation is an important mechanism involved in the silencing of TGF $\beta$ signalling in cancers [15-17]. Since we have not studied the mechanism underlying this gradual silencing of TGF $\beta$ signaling, it is inappropriate to reach a conclusion without understanding the exact mechanism.

Table 3. The pattern of protein expression of TGF $\beta$ members in PMD and OSCC samples. The mean expression is given as the mean log fold change with respect to normal.

\begin{tabular}{lccc}
\hline \multirow{2}{*}{ Protein } & \multicolumn{2}{c}{$\begin{array}{c}\text { Mean protein expression levels } \\
\text { (Compared to Normal) }\end{array}$} & p-Value (t-test) \\
\cline { 2 - 3 } & PMDs & OSCCs & \\
\hline pSMAD2 & -0.08059 & -0.1795 & 0.191 \\
pSMAD3 & -0.1347 & -0.1921 & 0.543 \\
SMAD2 & -0.0587 & -0.1840 & $\mathbf{0 . 0 1 5}$ \\
SMAD3 & -0.1240 & -0.1268 & 0.967 \\
SMAD4 & -0.0574 & -0.1147 & 0.356 \\
TGFBR1 & -0.0472 & -0.1205 & 0.221 \\
TGFBR2 & -0.0745 & -0.2822 & $\mathbf{0 . 0 1 4}$ \\
\hline
\end{tabular}


Also, the number of PMD cases used for the present analysis is comparatively low $(n=20)$. This necessitates evaluation in a larger cohort of PMDs.

More than $50 \%$ of the analyzed OSCC samples showed reduced expression level of TGF $\beta$-SMAD signaling members. Even though diminished levels of SMAD3, SMAD4 and TGFBR1 were found in a number of samples, the degree of downregulation was less as compared to SMAD2 and TGFBR2. In addition, only around $45 \%$ of samples showed robust R-SMAD phosphorylation, indicating disrupted SMAD signalling in OSCCs. Interestingly, we observed that the pSMAD/SMAD ratios are not significantly altered in PMDs and OSCCs. However, this result reflects the possibility of reduced SMAD phosphorylation due to reduction in the total

A)

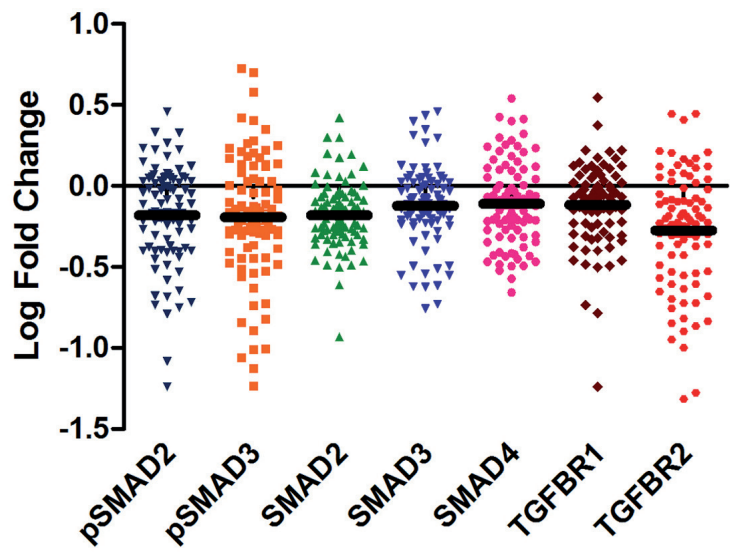

B)

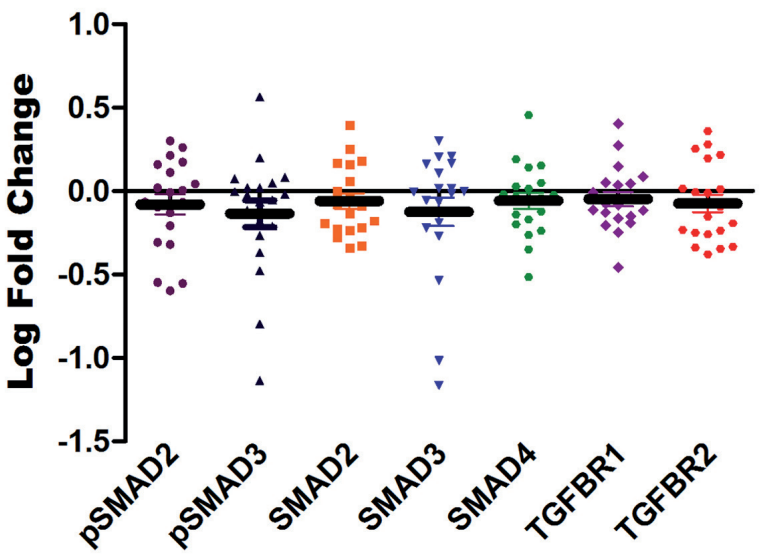

D)

TGFBR2 Protein Levels Vs Disease-free Survival

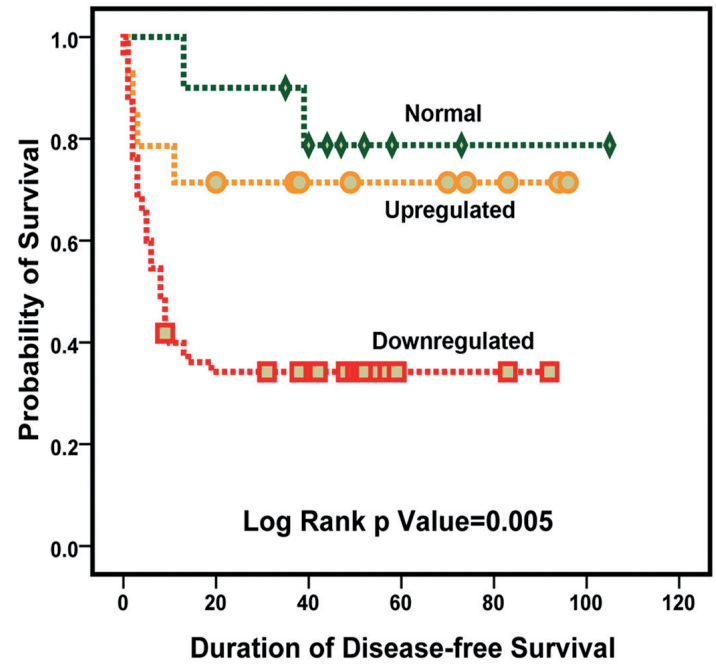

E)

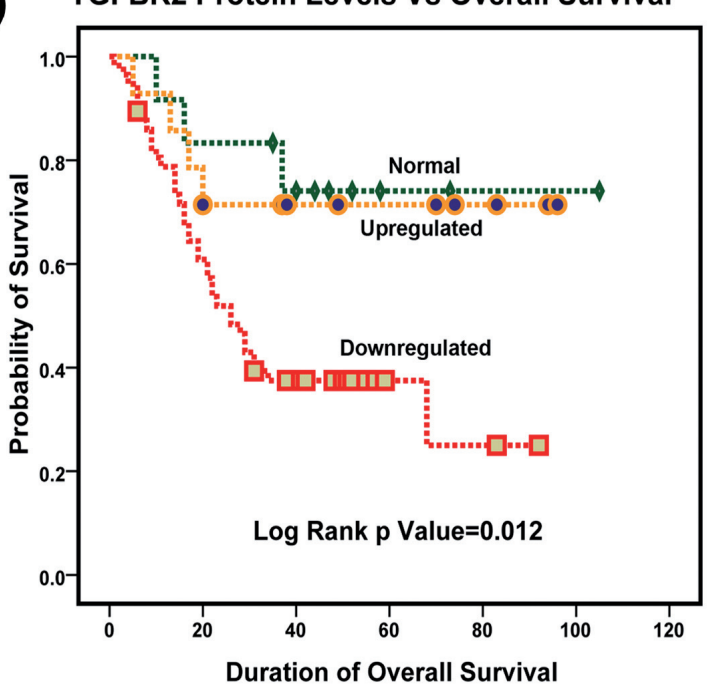

C)

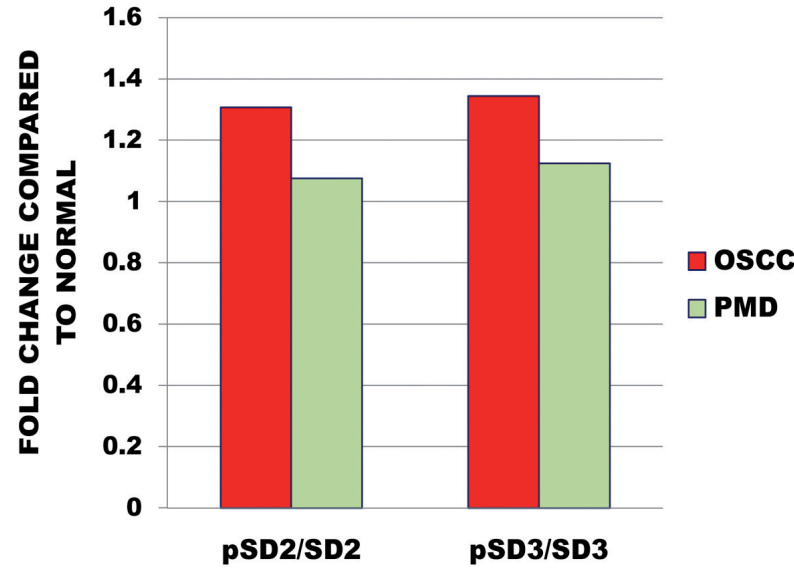

Figure 2. Expression pattern and prognostic association of TGF TGF $\beta$ pathway members in OSCCs. A \& B) Scatter plot diagram showing the expression pattern of analysed TGF $\beta$ pathway members in OSCCs and PMDs, respectively. C) The ratio of pSMAD to total SMAD in OSCCs and PMDs as compared to normal. D \& E) Prognostic significance of TGFBR2 protein levels in OSCCs. 
SMAD protein available. During the statistical analysis, this scenario can generate a pseudo-normal pSMAD/total SMAD ratio. However, this does not point towards an intact SMAD signalling cascade in malignancy. Our results are in agreement with Paterson's findings regarding the effect of reduced TGFBR2 levels in oral carcinoma [18]. His group showed the functional significance of the decrease in TGFBR2 expression by transfecting a dominant-negative TGFBR 2 construct into human oral carcinoma cell lines with normal TGFBR2 profile. Importantly, they found that the dnTGFBR2 causes decreased growth inhibition and SMAD binding activity whereas Fra-1 and collagenase- 1 expression remained unchanged. These results show that decreased TGFBR2 protein relative to TGFBR1 leads to selective gene regulation, with loss of growth inhibition but the transcription of AP-1-dependent genes that are involved in the regulation of the extracellular matrix remains unchanged. This study [18] could also explain the present result, because we also found a similar expression profile reduced TGFBR2 levels as compared to TGFBR 1 and decreased SMAD phosphorylation status in more than $50 \%$ of OSCC cases. However, another study on oral cancer demonstrated that the overexpression of TGF-b1 and IL- 6 were significantly correlated with the risk of lymph node involvement, disease recurrence and shorter survival in patients with advanced stage of disease [19]. But they have not looked on the expression status of other important downstream factors of the TGF-b1.

In a notable work, the expression of TGFBR2 was analyzed in the successive oncogenic stages of HNSCCs, from normal epithelium to dysplasia to carcinoma. Normal squamous epithelium and squamous epithelium near the tumors (adjacent normal) showed homogenous receptor expression of TGFBR2. Dysplastic epithelium and carcinoma in situ showed a mild decrease in receptor expression. Well-differentiated to moderately differentiated carcinomas showed heterogeneous expression of TGFBR 2 whereas poorly differentiated carcinomas were completely devoid of TGFBR2 [20]. This study had indicated that TGFBR 2 expression inversely correlates with disease aggressiveness and suggests that aberrant TGFBR2 expression is a contributing factor to the pathogenesis of HNSCCs. However, we could not find such a correlation between TGFBR2 protein levels and histopathology $(\mathrm{p}=0.484)$, even though a marginal association between low TGFBR2 expression and stage of disease was observed $(\mathrm{p}=0.063)$. This may be due to low sample size and has to be studied in a large population to confirm the present observation.

The clinico-pathological significance of SMAD4 and TGF $\beta 1$ in squamous cell carcinoma of the esophagus was evaluated in a notable study (21). Interestingly, patients with SMAD4 expression had a higher rate of early-stage carcinoma $(\mathrm{p}=<0.01)$ and fewer lymph node metastases $(\mathrm{p}=<0.01)$ compared to those with reduced SMAD4 expression. However, in multivariate analysis SMAD4 expression was not found to be independent and clinical factors such as patient age, depth of invasion, stage, and venous invasion were independent prognostic factors [21]. Further, a recent study demonstrated that decreased levels of both SMAD4 mRNA and protein in glioma compared to normal $(\mathrm{P}<0.001)$. There was a gradual decrease in SMAD4 expression levels from grade I to grade IV glioma [22]. The survival rate of SMAD4-positive patients was higher than that of SMAD4-negative patients and demonstrated that the loss of SMAD4 was a significant and independent prognostic indicator in glioma.

Similar to above mentioned studies, we also sought to determine the association between protein level TGF $\beta$-SMAD signalling alterations and clinical outcome in OSCCs. In ' $t$ '-test analysis, only TGFBR2 protein level was found to evolve as a promising prognostic factor in OSCCs. TGFBR2 downregulation was associated with poor disease-free survival as well as overall survival $(\mathrm{p}=0.005$ and $\mathrm{p}=0.012$ respectively). Even though SMAD2, pSMAD2 and pSMAD3 showed down regulation in a similar fashion, these were not associated with clinical outcome. TGFBR2 protein level was significant in multivariate analysis also for both DFS $(\mathrm{p}=0.020)$ and OS $\mathrm{p}=0.025$ ). Stage of the disease was found to be a significant factor for DFS $(\mathrm{p}=0.004)$ and OS $(\mathrm{p}=0.001)$ in this analysis. Further, TGFBR2 downregulated samples have more than two-fold increased risk for both poor DFS and OS. Notably, the final analysis of the results indicated the superior nature of TGFBR2 expression levels as compared to traditional clinical variables such as $\mathrm{T}$ and $\mathrm{N}$ status that are commonly used for predicting prognosis.

\section{Conclusion}

Even though the role of new markers in predicting the response and prognosis of head and neck cancer is still under development, it is becoming clear that individual markers are inadequate in constructing a prognostically meaningful tumor profile for each patient. Hence, it is suggested to use a number of well-characterized markers acting in unrelated cellular pathways for successfully seperate prognostic patient categories of greater utility than traditional TNM staging. Our results clearly indicate that those oral cancers that show TGFBR2 downregulation comprise a different group with more aggressiveness. Hence, it could be beneficial for the OSCC patients if TGFBR2 expression pattern is also considered during treatment to define different prognostic groups so as to administer optimum treatment.

Supplementary information is available in the online version of the paper.

Acknowledgments: This study was supported by the Department of Biotechnology (DBT), Govt. of India through grant No.BT/PR10973/GBD/27/130/2008. Mr. Sivadas is a senior research fellow under the UGC-SRF programme of University Grants Commission (UGC), Govt. of India. Authors are thankful to Dr. Sathyan KM, Dan Foltz's Lab, Department of Biochemistry \& Molecular Genetics, University of Virginia, USA for his support and suggestions. 


\section{References}

[1] SHAH JP, JOHNSON NW, BATSAKIS JG, editors. Oral Cancer. London: Taylor and Francis Group. 2003. pp. 496.

[2] BAGAN J, SARRION G, JIMENEZ Y. Oral Cancer: Clinical Features. Oral Oncol 2010; 46: 414-417. http://dx.doi. org/10.1016/j.oraloncology.2010.03.009

[3] BOYLE P, LEVIN B, editors. World Cancer Report. International Agency for Research on Cancer, France: IARC Press, Lyon Cedex 08. 2008.

[4] CURADO MP, EDWARDS B, SHIN HR, STORM H, FERLAY J, et al. Cancer incidence in five continents, volume IX. International Agency for Research on Cancer, France: IARC Press, Lyon Cedex 08. 2009.

[5] MUWONGE R, RAMADAS K, SANKILA R, THARA S, THOMAS G, et al. Role of tobacco smoking, chewing and alcohol drinking in the risk of oral cancer in Trivandrum, India: A nested case-control design using incident cancer cases. Oral Oncol 2008; 44: 446-454. http://dx.doi.org/10.1016/j. oraloncology.2007.06.002

[6] HERRERO R, CASTELLSAGUE X, PAWLITA M, LISSOWSKA J, KEE F, et al. Human papillomavirus and oral cancer: the International Agency for Research on Cancer multicenter study. J Natl Cancer Inst. 2003; 95: 1772-1783. http://dx.doi. org/10.1093/jnci/djg107

[7] da SILVA SD, HIER M, MLYNAREK A, KOWALSKI LP, ALAOUI- JAMALI MA. Recurrent oral cancer: current and emerging therapeutic approaches. Front Pharmacol 2012; 3: 149. http://dx.doi.org/10.3389/fphar.2012.00149

[8] SHI Y, MASSAGUE J. Mechanisms of TGF-beta signaling from cell membrane to the nucleus. Cell 2003; 113: 685-700. http://dx.doi.org/10.1016/S0092-8674(03)00432-X

[9] SEOANE J. Escaping from the TGFbeta anti- proliferative control. Carcinogenesis 2006; 27: 2148-2156. http://dx.doi. org/10.1093/carcin/bgl068

[10] MASSAGUE J. TGF-beta signal transduction. Annu Rev Biochem 1998; 67: 753-791. http://dx.doi.org/10.1146/annurev. biochem.67.1.753

[11] KIM IY, AHN HJ, ZELNER DJ, SHAW JW, LANG S, et al. Loss of expression of transforming growth factor beta type I and type II receptors correlates with tumor grade in human prostate cancer tissues. Clin Cancer Res 1996; 2: $1255-1261$.

[12] PINDBORG JJ, REICHART PA, SMITH CJ. WHO: histological typing of cancer and pre-cancer of the oral mucosa. USA: Springer-Verlag; 1997 p. 11-34
[13] MURALI A, SAILASREE R, SEBASTIAN P, KUMAR RR, VARGHESE BT, et al. Loss of heterozygosity of D9S162: Molecular predictor for treatment response in oral carcinoma. Oral Oncol 2011; 47: 571-576. http://dx.doi.org/10.1016/j. oraloncology.2011.04.009

[14] SIVADAS VP, GEORGE NA, KATTOOR J, KANNAN S. Novel mutations and expression alterations in SMAD3/ TGFBR2 genes in oral carcinoma correlate with poor prognosis. Genes Chromosomes Cancer 2013; 52 : 1042-1052. http://dx.doi.org/10.1002/gcc.22099

[15] ZHANG HT, CHEN XF, WANG MH, WANG JC, QI QY, et al. Defective expression of transforming growth factor beta receptor type II is associated with CpG methylated promoter in primary non-small cell lung cancer. Clin Cancer Res 2004; 10: 2359-2367. http://dx.doi.org/10.1158/1078-0432.CCR-0959-3

[16] ZHAO H, SHIINA H, GREENE KL, LI LC, TANAKA Y, et al. CpG methylation at promoter site - 140 inactivates TGFbeta2 receptor gene in prostate cancer. Cancer 2005; 104: 44-52. http://dx.doi.org/10.1002/cncr.21135

[17] YAMASHITA S, TAKAHASHI S, MCDONELL N, WATANABE N, NIWA T, et al. Methylation silencing of transforming growth factor-beta receptor type II in rat prostate cancers. Cancer Res 2008; 68: 2112-2121. http://dx.doi. org/10.1158/0008-5472.CAN-07-5282

[18] PATERSON IC, MATTHEWS JB, HUNTLEY S, ROBINSON CM, FAHEY M, et al. Decreased expression of TGF-beta cell surface receptors during progression of human oral squamous cell carcinoma. J Pathol 2001; 193: 458-467. http:// dx.doi.org/10.1002/1096-9896(2000)9999:9999<::AIDPATH822>3.0.CO;2-V

[19] CHEN MF, WANG WH, LIN PY, LEE KD, CHEN WC. Significance of the TGF- $\beta 1 /$ IL- 6 axis in oral cancer. Clin Sci (Lond). 2012; 122: 459-72. http://dx.doi.org/10.1042/ CS20110434

[20] MURO-CACHO CA, ANDERSON M, CORDERO J, MUNOZ-ANTONIA T. Expression of transforming growth factor $\beta$ type II receptors in head and neck squamous cell carcinoma. Clin Cancer Res 1999; 5: 1243-1248.

[21] NATSUGOE S, XIANGMING C, MATSUMOTO M, OKUMURA H, NAKASHIMA S, et al. Smad4 and transforming growth factor betal expression in patients with squamous cell carcinoma of the esophagus. Clin Cancer Res 2002; 8: 1838-1842.

[22] HE SM, ZHAO ZW, WANG Y, ZHAO JP, WANG L, et al. Reduced expression of SMAD4 in gliomas correlates with progression and survival of patients. J Exp Clin Cancer Res 2011; 30: 70. http://dx.doi.org/10.1186/1756-9966-30-70 